\title{
A PRODUÇ̃̃O DO CONHECIMENTO SOBRE ADOLESCÊNCIA NA ENFERMAGEM: PERÍODO 1983 A 1996
}

\author{
Maria Aparecida Tedeschi Cano* \\ Maria das Graças Carvalho Ferriani** \\ Ana Cristina Alves*** \\ Cristina Yuri Nakata***
}

CANO, M.A.T.; FERRIANI, M.das G.C.; ALVES, A.C.; NAKATA, C.Y. A produção do conhecimento sobre adolescência na enfermagem: período 1983 a 1996. Rev.latino-am.enfermagem, Ribeirão Preto, v. 6, n. 1, p. 9197, janeiro 1998.

Nos últimos dez anos, a adolescência tem sido objeto de estudo de diversos pesquisadores e considerada por Organizações Nacionais e Internacionais uma faixa etária prioritária para ações de promoção, prevenção e proteção à saúde. Vários fatores contribuirram para essa preocupação com a adolescência, entre eles podemos destacar a importância desse contingente populacional, que constitui $23 \%$ da população mundial, além de questões ligadas à gravidez precoce, à AIDS e às drogas. Como enfermeiras pesquisadoras, interessamo-nos por conhecer e caracterizar, quantitativa e qualitativamente, a produção cientifica sobre adolescência em enfermagem, utilizando como fonte de coleta de dados periódicos nacionais específicos de enfermagem. Os dados obtidos nesse estudo nos mostram que a área da adolescência ainda é pouco explorada pela enfermagem e os aspectos mais pesquisados são os relacionados à sexualidade.

UNITERMOS: adolescência, enfermagem

\section{INTRODUÇÃO}

A adolescência, nas últimas décadas, vem sendo objeto de estudos de diferentes pesquisadores (OSÓRIO, 1992; ARATANGY, 1995; VITIELLO, 1988; TIBA, 1986; FERRIANI et al., 1992). A importância que a adolescência assumiu nesse período está ligada a diversos fatores, entre eles a maior liberdade sexual, decorrente de diversos movimentos sociais, como a Revolução Sexual iniciada na década de 50 e o movimento feminista; o aparecimento da AIDS e a elevação do consumo de drogas. Cabe ressaltar, também, a importância numérica dos adolescentes que representam $23,4 \%$ da população brasileira e $30 \%$ da população mundial (BRASIL, M.S., 1989).

Segundo VITIELLO (1988), alguns autores consideram, em termos cronológicos, que a fase da adolescência inicia-se por volta dos 11 anos e termine aos 20 anos, enquanto outros autores propõem faixas etárias diferentes. VITIELLO considera muito difícil fixar limites para a adolescência, uma vez que esta fase sofre influências sociais, culturais, familiares e pessoais.

O Programa de Saúde do Adolescente - PROSAD - estabeleceu para a adolescência, a faixa etária que vai dos 10 aos 19 anos (BRASIL, M.S., 1989).

Para CAVALCANTI (1988), a adolescência é uma fase de crescimento biopsicossocial que está entre a infância e a idade adulta. "O crescer, para que seja uma experiência equilibrada de vida, deve implicar num crescimento solidário biológico, sociocultural e psicológico" (p. 6).

VITIELLO (1988) acrescenta que, muitas vezes, as manifestações físicas precedem as de natureza psicossocial e o que se tem é um indivíduo com o corpo apresentando características sexuais secundárias, mas com atitudes infantis ou ao contrário, indivíduos rebeldes, contestadores, em crises existenciais, com um corpo infantil. Para esse autor, em ambas as situações, a adolescência já começou.

Em OSÓRIO (1992) encontramos que a

\footnotetext{
* Professora Doutora do Departamento de Enfermagem Materno-Infantil e Saúde Pública da Escola de Enfermagem de Ribeirão Preto da Universidade de São Paulo

** Professora Associada do Departamento de Enfermagem Materno-Infantil e Saúde Pública da Escola de Enfermagem de Ribeirão Preto da Universidade de São Paulo

*** Bolsistas de Iniciação Científica do CNPq
} 
adolescência é um marco na vida das pessoas e o momento crucial do desenvolvimento, na qual o indivíduo, além das transformações físicas, está construindo, ao nível do emocional e psicológico, sua imagem corporal definitiva e estruturando sua personalidade.

Para ERICKSON (1972), na adolescência o indivíduo vive um momento de "crise", mas no sentido positivo, ou seja, está adquirindo novos conhecimentos, se reestruturando, amadurecendo. Aponta ainda, o autor, que esse período é necessário, podendo resultar em um ser mais saudável, maduro e preparado para enfrentar a vida adulta.

Segundo o Programa de Saúde do Adolescente PROSAD, o adolescente é um ser idealista, curioso, contestador e esses sentimentos despertam nele uma necessidade de desafio que, associado à falta de experiência e vivência anterior, pode levá-lo a condutas de risco. A mudança em seu estilo de vida o torna suscetível à violência, aos acidentes, uso de drogas, gravidez indesejada, entre outros (BRASIL, M.S., 1989).

É a partir das últimas décadas que a adolescência passou a ser vista de maneira mais global e as mudanças físicas, psíquicas e sociais se configuram em um quadro de vulnerabilidade aos agravos sociais e, portanto, os adolescentes passam a merecer maior atenção em termos de saúde.

Através das citações dos vários autores podemos observar que o adolescente está vivendo um momento peculiar da fase de desenvolvimento do ser humano, com várias modificações ocorrendo ao mesmo tempo, o que o torna um ser vulnerável e necessitado de apoio, proteção e orientação.

Enquanto profissionais de saúde, estamos frequentemente atuando junto a adolescentes nos hospitais, na rede básica de saúde ou nas escolas e, a partir dessa vivência, vimos a necessidade e a importância de os enfermeiros ampliarem seus conhecimentos sobre esta faixa etária; para tanto buscamos conhecer, na literatura nacional, a produção científica dos enfermeiros sobre adolescência, tendo por objetivos:

- realizar um levantamento sobre a produção do conhecimento dos enfermeiros sobre a adolescência, no período de 1983 a 1996 ;

- identificar os aspectos da adolescência que são mais enfocados pelos enfermeiros;

- identificar os aspectos da adolescência que ainda não foram estudados;
- qual a contribuição das pesquisas na assistência de enfermagem ao adolescente.

\section{METODOLOGIA}

Este trabalho utilizou como fonte de dados os ANAIS de Congressos Brasileiros de Enfermagem e periódicos nacionais de enfermagem publicados no período compreendido entre 1983 a 1996.

Os dados foram coletados mediante consulta aos periódicos nacionais encontrados, de onde foram selecionados todos os artigos pertinentes ao tema "adolescência". Realizada a seleção, utilizou-se um instrumento de coleta de dados que continha os seguintes itens: a) título do trabalho; b) autor responsável pelo trabalho; c) temática abordada; d) tipo de abordagem: quantitativo e/ou qualitativo.

As revistas consultadas foram: a) Revista Brasileira de Enfermagem; b) Revista Paulista de Enfermagem; c) Revista Gaúcha de Enfermagem; d) Revista da Escola de Enfermagem da USP; e) Enfermagem Moderna; f) Revista Latino-Americana de Enfermagem; g) Revista Paulista de Hospitais; h) Revista Baiana de Enfermagem; i) Anais de Enfermagem. Cabe ressaltar que a Revista Paulista de Hospitais foi incluída por ser um periódico que publica um número considerável de artigos produzidos por enfermeiros. Quanto à RevistaLatino Americana de Enfermagem sua publicação iniciou-se em 1993.

Primeiramente foi realizado um levantamento bibliográfico através do LILACS* mas não foi possível a obtenção dos dados solicitados, uma vez que muitas revistas de enfermagem ainda não são indexadas. Optouse, então, pelo levantamento bibliográfico periódico por periódico. Infelizmente muitas revistas estavam com numeração incompleta e foi necessário buscar, através do COMUT**, na Biblioteca Central do Campus da Universidade de São Paulo, em Ribeirão Preto, os números não encontrados. Alguns foram localizados enquanto que outros não.

\section{RESULTADOS E DISCUSSÃO}

Após a coleta de dados, os resultados foram organizados em tabelas.

\footnotetext{
* LILACS - Literatura Latino Americana e do Caribe em Ciências da Saúde desde 1981

** COMUT - Programa de Comutação Bibliográfica
} 
Tabela 1 - Distribuição dos Periódicos e Anais de Enfermagem, segundo o ano de publicação (1983 a 1996)

\begin{tabular}{l|c|c|c|c|c|c|c|c|c|c|c|c|c|c|c}
\hline $\begin{array}{c}\text { Anos } \\
\text { Periódicos }\end{array}$ & $\mathbf{8 3}$ & $\mathbf{8 4}$ & $\mathbf{8 5}$ & $\mathbf{8 6}$ & $\mathbf{8 7}$ & $\mathbf{8 8}$ & $\mathbf{8 9}$ & $\mathbf{9 0}$ & $\mathbf{9 1}$ & $\mathbf{9 2}$ & $\mathbf{9 3}$ & $\mathbf{9 4}$ & $\mathbf{9 5}$ & $\mathbf{9 6}$ & To tal \\
\hline $\begin{array}{l}\text { Rev. Bras. } \\
\text { Enfermagem }\end{array}$ & 3 & 3 & 3 & 2 & 3 & 3 & 1 & 1 & 3 & 3 & 3 & 4 & 4 & $\#$ & 36 \\
\hline $\begin{array}{l}\text { Rev. Paul. } \\
\text { Enfermagem }\end{array}$ & 5 & 4 & 4 & 4 & 1 & 2 & $*$ & 3 & 3 & 3 & 2 & 1 & 1 & 1 & 34 \\
\hline $\begin{array}{l}\text { Rev. Baiana } \\
\text { Enfermagem }\end{array}$ & $\#$ & $\#$ & 2 & 2 & 1 & 1 & $\#$ & $\#$ & $\#$ & 1 & 2 & 1 & 1 & 1 & 12 \\
\hline $\begin{array}{l}\text { Rev. Gaúcha } \\
\text { Enfermagem }\end{array}$ & 2 & 2 & 2 & 2 & 2 & 2 & 2 & 2 & 1 & $\#$ & $\#$ & 1 & 1 & 1 & 20 \\
\hline $\begin{array}{l}\text { Rev. Escola } \\
\text { Enf. USSP }\end{array}$ & 3 & 3 & 3 & 3 & 3 & 3 & 3 & 3 & 3 & 4 & $\#$ & 3 & 3 & 3 & 40 \\
\hline $\begin{array}{l}\text { Enfermagem } \\
\text { Modema }\end{array}$ & 4 & 4 & 3 & $\#$ & $\#$ & $\#$ & $\#$ & $\#$ & $\#$ & $\#$ & $\#$ & $\#$ & $\#$ & $\#$ & 11 \\
\hline $\begin{array}{l}\text { Rev. Paul. } \\
\text { de Hospitais }\end{array}$ & 6 & 6 & 6 & 4 & 4 & 4 & 3 & 3 & 3 & 2 & 2 & 1 & 1 & 1 & 46 \\
\hline $\begin{array}{l}\text { Rev. Latino- } \\
\text { Americ. Enf. }\end{array}$ & $*$ & $*$ & $*$ & $*$ & $*$ & $*$ & $*$ & $*$ & $*$ & $*$ & 3 & 2 & 2 & 4 & 11 \\
\hline $\begin{array}{l}\text { Anais de } \\
\text { Enfermagem }\end{array}$ & 1 & 1 & 1 & 1 & 1 & $\#$ & 1 & $\#$ & 1 & 1 & 1 & 1 & 1 & $\#$ & 11 \\
\hline \begin{tabular}{l} 
TOTAL \\
\hline
\end{tabular}
\end{tabular}

Obs: * Não houve publicação

\# Os periódicos não foram encontrados

A Tabela 1 refere-se à distribuição dos periódicos Foram encontradas 210 revistas e 11 Anais que continham nacionais, segundo o ano de publicação (1983 a 1996). um total de 73 artigos relativos à adolescência.

Tabela 2 - Distribuição dos Artigos sobre Adolescência encontrados nos Periódicos nos anos de Publicação (1983 - 1996)

\begin{tabular}{l|c|c|c|c|c|c|c|c|c|c|c|c|c|c|c}
\hline $\begin{array}{c}\text { Anos } \\
\text { Periódicos }\end{array}$ & $\mathbf{8 3}$ & $\mathbf{8 4}$ & $\mathbf{8 5}$ & $\mathbf{8 6}$ & $\mathbf{8 7}$ & $\mathbf{8 8}$ & $\mathbf{8 9}$ & $\mathbf{9 0}$ & $\mathbf{9 1}$ & $\mathbf{9 2}$ & $\mathbf{9 3}$ & $\mathbf{9 4}$ & $\mathbf{9 5}$ & $\mathbf{9 6}$ & T0 tal \\
\hline $\begin{array}{l}\text { Rev. Bras. } \\
\text { Enfermagem }\end{array}$ & 1 & 1 & 3 & 1 & 0 & 1 & 2 & 0 & 1 & 2 & 1 & 0 & 0 & $\#$ & 13 \\
\hline $\begin{array}{l}\text { Rev. Paul. } \\
\text { Enfermagem }\end{array}$ & 2 & 2 & 2 & 0 & 0 & 0 & $*$ & 0 & 0 & 1 & 0 & 0 & 0 & 0 & 7 \\
\hline $\begin{array}{l}\text { Rev. Baiana } \\
\text { Enfermagem }\end{array}$ & & & 1 & 0 & 0 & 0 & & & & 1 & 0 & 0 & 0 & 0 & 2 \\
\hline $\begin{array}{l}\text { Rev. Gaúcha } \\
\text { Enfermagem }\end{array}$ & 0 & 1 & 1 & 0 & 0 & 1 & 1 & 1 & 1 & & & 0 & 4 & 0 & 10 \\
\hline $\begin{array}{l}\text { Rev. Escola } \\
\text { Enf. USP }\end{array}$ & 0 & 0 & 0 & 0 & 0 & 0 & 0 & 0 & 0 & 0 & & 0 & 0 & 0 & 0 \\
\hline $\begin{array}{l}\text { Enfermagem } \\
\text { Modema }\end{array}$ & 0 & 0 & 0 & & & & & & & & & $\#$ & $\#$ & $\#$ & 0 \\
\hline $\begin{array}{l}\text { Rev. Paul. } \\
\text { de Hospitais }\end{array}$ & 0 & 0 & 1 & 1 & 0 & 1 & 0 & 2 & 1 & 0 & 0 & 1 & 0 & 0 & 7 \\
\hline $\begin{array}{l}\text { Rev. Latino- } \\
\text { Americ. Enf. }\end{array}$ & $*$ & $*$ & $*$ & $*$ & $*$ & $*$ & $*$ & $*$ & $*$ & $*$ & 0 & 0 & 1 & 3 & 4 \\
\hline $\begin{array}{l}\text { Anais de } \\
\text { Enfermagem }\end{array}$ & 0 & 0 & 1 & 1 & 4 & & 0 & & 7 & 5 & 0 & 1 & 11 & $\#$ & 30 \\
\hline \begin{tabular}{l} 
ToTAL \\
\hline
\end{tabular}
\end{tabular}

Obs: 0 - Não foi encontrado artigo sobre adolescência

- As revistas não foram encontradas

* - Não houve publicação

\# - 1986 - Ano Internacional da Juventude 
$\mathrm{Na}$ Tabela 2, que se refere à distribuição dos artigos sobre adolescência encontrados nos periódicos e Anais, no período de 1983 a 1996, verifica-se que os Anais de Enfermagem apresentaram um maior número de artigos sobre o tema, entretanto é preciso ressaltar que nesta forma de publicação aparecem apenas os resumos das investigações. De qualquer forma, percebe-se que há interesse dos enfermeiros pelas questões da adolescência, uma vez que estão levando para os Congressos suas pesquisas na área. $\mathrm{O}$ importante é que posteriormente os enfermeiros encaminhem essas pesquisas também para as revistas, atingindo um maior número de profissionais interessados nesse tema.

De acordo com esta tabela, observa-se que, entre as revistas pesquisadas, a Revista Brasileira de Enfermagem é a que apresenta um maior número de artigos publicados. Esse fato talvez possa ser explicado por ser este um periódico tradicional, conhecido em âmbito nacional pelos enfermeiros e vinculado à Associação Brasileira de Enfermagem.

Ainda na Tabela 2, imaginou-se que no ano de 1986, considerado pela Organização Mundial de Saúde como o Ano Internacional da Juventude, haveria um número significativo de artigos, no entanto apenas três trabalhos foram publicados.

Apesar da importância que a adolescência vem assumindo nas últimas décadas e por ser um tema freqüentemente abordado na literatura por pesquisadores de diferentes áreas do conhecimento, na enfermagem ainda existe escassez de artigos publicados.

UBEDA (1996) realizou um levantamento de dissertações e teses de enfermagem na "área de adolescência", nos catálogos do Centro de Estudos e Pesquisas em Enfermagem - CEPEN, de 1979 ( $1^{\circ}$ volume) a 1996 (último volume), mostrando que a produção científica ainda é muito pequena. Foram identificados 14 trabalhos, todos eles em dissertações de mestrado. Acrescenta, ainda, a autora que as temáticas mais pesquisadas abordam fatos e conhecimentos que afetam a vida do adolescente, com ênfase nas questões relacionadas à sexualidade, gravidez, drogas, álcool e fumo, seguida da assistência à saúde do adolescente no que diz respeito à gravidez, parto e puerpério.

Tabela 3 - Distribuição das temáticas mais abordadas sobre adolescência nos artigos analisados (1983 - 1996)

\begin{tabular}{l|c|c}
\hline \multicolumn{1}{c|}{ TEMÁTICAS } & NÚMERO & FREQÜÊNCIA (\%) \\
\hline Educação Sexual & 15 & 20,54 \\
\hline Gravidez & 14 & 19,17 \\
\hline Saúde Mental & 11 & 15,06 \\
\hline Anticoncepção & 8 & 6,84 \\
\hline DST/AIDS & 5 & 4,10 \\
\hline Educação em Saúde & 3 & 6,84 \\
\hline Assistência a patologias & 5 & 4,10 \\
\hline Tóxicos & 3 & 2,73 \\
\hline Parto/Puerpério & 2 & 1,36 \\
\hline Mães adolescentes & 1 & 1,36 \\
\hline Teorias de Enfermagem & 1 & 1,36 \\
\hline Familia/Adolescente & 1 & 1,36 \\
\hline Meninos de rua & 1 & 1,36 \\
\hline Mortalidade & 1 & 1,36 \\
\hline Participação Social & 1 & 1,36 \\
\hline Reciclagem profissional & 1 & $\mathbf{1 0 0}$ \\
\hline ToTAL & 73 & \\
\hline
\end{tabular}

Na tabela 3, verifica-se que a temática mais abordada foi Educação Sexual. Segundo CONCEIÇÃO (1988), "a educação sexual realizada em nossa sociedade, tem como objetivo uma ostensiva repressão à sexualidade humana. As regras codificadas pelo sistema social vigente aceitam o exercício da sexualidade somente entre casais legitimamente unidos em matrimônio, e, mesmo assim, esse deveria limitar-se à reprodução, sendo condenado 
quando praticado na busca do prazer". (p. 71).

Após a revolução sexual, ocorrida nos anos 60, que contestava as normas vigentes, afirma a autora que o sexo passou a ser utilizado com os mais diferentes propósitos: para agredir o sistema conservador, com finalidade exclusivamente econômica, praticado por adolescentes despreparados para a atividade sexual, ou explorado com sensacionalismo e vulgaridade pelos meios de comunicação.

Ainda segundo CONCEIÇÃO (1988), para que o indivíduo viva uma vida sexual livre de tabus e preconceitos, há necessidade de transformações no sistema educacional e na própria sociedade. "Um sistema de educação autoritária e opressora não oferece oportunidade de crescimento ao indivíduo como pessoa livre e capaz de escolher o seu destino. Este sistema educacional não é compatível com o exercício sadio da sexualidade, que é a expressão livre e natural do relacionamento humano" (p. 72).

Seguindo esta linha de raciocínio, pensamos que a educação sexual bem conduzida pode ajudar os jovens a melhorar sua qualidade de vida, tornando-os mais tranqüilos em relação à sua sexualidade.

Para PARKER (1991) cada vez mais a sexualidade tem se tornado tema de discussões e debates na sociedade brasileira, uma vez que controvérsias como o aborto, os direitos das minorias sexuais e, mais recentemente, a alarmante propagação da AIDS se colocaram no centro da atenção pública na vida contemporânea.

A segunda temática mais abordada foi gravidez na adolescência. De acordo com projeções do IBGE, $20 \%$ das crianças nascidas vivas no Brasil, são filhos de mães adolescentes. Nos Estados Unidos esse índice cai para $16 \%$ e no Canadá é de 9,5\% (Folha de São Paulo, 1991).

Dados semelhantes foram encontrados por LOPEZ et al. (1989) em um município da grande São Paulo, onde $22,2 \%$ da população de gestantes era constituída de adolescentes.

Muitos autores acreditam que a adolescente não está emocionalmente preparada para enfrentar a gravidez, o parto e o processo de criação de um filho. A gravidez acontece geralmente de forma inesperada, trazendo consigo uma série de problemas que vão interferir no desenvolvimento da jovem, como por exemplo, a não aceitação da família e as restrições sociais e econômicas, principalmente quando deixa de estudar para assumir a maternidade, perdendo, com isso, a possibilidade de ascensão social através dos estudos (ARATANGY, 1995; BARROSO \& BRUSCHINI, 1991; SEXUALIDADE, 1993).

Esses autores relatam também que o uso de contraceptivos pelas adolescentes é baixo, sendo que algumas desconhecem a anatomia do próprio corpo, assim como a fisiologia da concepção e reprodução. Por outro lado, acrescentam que, mesmo entre as adolescentes mais informadas, usar contraceptivos significa assumir socialmente sua vida sexual.

Para OSÓRIO (1992), se a anticoncepção tem seus inconvenientes, ou até mesmo contra-indicações na adolescência, a interrupção de uma gravidez indesejada, através do aborto, traz maiores riscos físicos e emocionais que a anticoncepção. Os adultos precisam permitir que os adolescentes tenham, não só acesso à informação, mas também ao uso dos contraceptivos. "Prevenir uma concepção indesejada e um aborto conseqüente é uma responsabilidade de todos nós: pais, educadores e médicos" (p. 43).

A outra temática abordada refere-se à saúde mental, englobando assistência emocional ao adolescente, grupos de adolescentes, entre outros. Para OUTEIRAL (1994), é necessário entender que na adolescência normal há momentos progressivos e regressivos do emocional, em função de tensões internas e/ou externas que o adolescente enfrenta. As tensões internas são modificações próprias da adolescência. As tensões externas referem-se à relação do adolescente com o meio familiar e social. Os adolescentes, de uma maneira geral, têm dificuldade de expressar seus sentimentos em palavras e o fazem, preferencialmente, através de atitudes. Aos poucos vão se organizando mentalmente e conseguem expressar um discurso verbal coerente com seus sentimentos e idéias.

Concordamos com os autores citados e acreditamos que seja fundamental para o enfermeiro conhecer esses aspectos da adolescência e estar preparado para participar da tarefa educativa, apoiando, discutindo e participando da realidade em que o adolescente vive.

Um tema pouco abordado pelos autores foi Tóxicos na Adolescência, o que, de certa forma, nos preocupa, uma vez que este é um problema bastante freqüente em nossa sociedade.

FERRIANI et al. (1992) nos mostram essa realidade ao realizarem um levantamento sobre o conhecimento de alunos de $1^{\circ}$ grau, das escolas públicas de Ribeirão Preto - SP, sobre drogas, no qual concluíram que todos os alunos, independente do nível sócioeconômico, possuem algum conhecimento a respeito do tema.

Segundo CANELLA (1988) é muito "comum na adolescência o uso de soluções mágicas para os problemas, o que acaba por facilitar as experiências com os tóxicos, os permitidos e os proibidos, na tentativa de alcançar a independência e a sedimentação da individualidade. A civilização de consumo aproveita-se da vulnerabilidade do jovem dirigindo a ele suas mensagens publicitárias, criando valores e induzindo ao 
consumo do tabaco e do álcool”. O consumo de tóxicos na adolescência geralmente declina ou desaparece à medida que o jovem amadurece" (p. 63).

Ainda segundo o autor, é importante não confundir a experimentação de drogas movida pela curiosidade e caracterizada pelo seu uso intermitente ou doses moderadas com a necessidade compulsiva de consumo, que caracteriza a dependência.

Através da análise dos artigos, verificamos que a contribuição das pesquisas em enfermagem estão mais direcionadas para as questões da Educação Sexual, ciclo gravídico-puerperal e anticoncepção, como partes da sexualidade, oferecendo subsídios para os enfermeiros que atuam na área de ginecologia e obstetrícia, saúde da mulher e saúde escolar. Esta realidade talvez possa ser explicada pela importância assumida por essas questões na sociedade contemporânea.

UBEDA (1996) é de opinião que os "enfermeiros ainda não apresentam uma preocupação concreta em relação à adolescência e nem estão totalmente inseridos na prática de atendimento integral à saúde do adolescente". Concordamos com a autora e pensamos que esta situação esteja relacionada à deficiência da formação dos enfermeiros na área de adolescência, uma vez que só recentemente esse conteúdo passou a integrar o currículo mínimo de Enfermagem, aprovado pela Portaria MEC 1721/94, que estabelece que os cursos de graduação deverão contar com o ensino da assistência de enfermagem ao adolescente, nos níveis individual e coletivo.

Talvez esse fato também explique porque temas como violência doméstica e urbana, oncologia, vacinação e muitos outros, ainda não apareçam como problemas de investigação nas pesquisas dos enfermeiros sobre adolescência.

De uma maneira geral, os artigos produzidos utilizaram uma metodologia quantitativa para abordar a temática adolescência.

Tabela 4 - Distribuição do primeiro autor dos artigos segundo sua área de atuação (1983 - 1996)

\begin{tabular}{l|c|c}
\hline \multicolumn{1}{c|}{ CATEGORIA } & TOTAL & FREQÜÊNCIA (\%) \\
\hline Docente de Enfermagem & 30 & 41,09 \\
\hline Enfermeira de campo & 10 & 13,69 \\
\hline Aluno de Graduação & 5 & 6,84 \\
\hline Aluno de Pós-Graduação & 4 & 5,47 \\
\hline Autornão identificado & 4 & 5,47 \\
\hline $\begin{array}{l}\text { Enfermeiros sem } \\
\text { explicitação da categoria }\end{array}$ & 18 & 24,65 \\
\hline Outros profissionais & 2 & 2,73 \\
\hline TOTAL & $\mathbf{7 3}$ & $\mathbf{1 0 0}$
\end{tabular}

Em relação à Tabela 4, verifica-se que a categoria de enfermagem que mais publicou artigos sobre o tema adolescência foi a dos docentes. Isto pode-se justificar pelas exigências que a Universidade faz para que os mesmos realizem pesquisa científica. O enfermeiro assistencial, distante da Universidade, publica poucos trabalhos científicos, às vezes por falta de incentivo e outras por desconhecimento dos princípios de realização de um trabalho científico ou até mesmo por não dar importância à divulgação de trabalhos que são realizados por eles no cotidiano dos campos de atuação prática. Cabe enfatizar a presença de alunos de graduação desenvolvendo trabalhos de pesquisa na área de adolescência.

\section{CONSIDERAÇÕES FINAIS}

Como verificamos na Introdução desse trabalho, vários autores colocam que o adolescente está vivendo um momento peculiar da fase de desenvolvimento do ser humano, com várias modificações ocorrendo ao mesmo tempo, o que o torna um ser vulnerável e necessitado de apoio, proteção e orientação.

Pensamos que os jovens brasileiros de hoje, têm que "lidar" não apenas com seus problemas físicos e emocionais, mas também com a crise social e econômica pela qual passa a sociedade brasileira.

Frente a essa realidade, os enfermeiros precisam buscar aprofundar conhecimentos através da pesquisa sobre essa etapa da vida do ser humano, dentro de um contexto sócio-político e cultural. Desta forma poderão planejar uma assistência de qualidade, voltada para as necessidades dessa população. É importante, ainda, que outros aspectos da adolescência, além da sexualidade e assistência à saúde mental, sejam melhor explorados nas pesquisas.

Os enfermeiros de campo, que vivem em contato com adolescentes nas unidades de internação, na rede básica de saúde ou nas escolas, também precisam realizar pesquisas e publicar os conhecimentos adquiridos em sua prática diária.

Além da apresentação dos estudos realizados na área de adolescência em eventos científicos, os enfermeiros precisam divulgar esses estudos através da publicação em revistas de enfermagem, atingindo dessa forma um maior número de profissionais uma vez que, as revistas de circulação nacional "funcionam como ensino à distância".

Com este trabalho buscamos conhecer como se encontrar a pesquisa sobre adolescência na enfermagem e verificamos que esta é uma área ainda a ser melhor explorada pelos enfermeiros.

O enfermeiro como educador tem uma importante contribuição na formação e orientação dos adolescentes principalmente em nível de promoção à saúde. 
In the last ten years adolescence has been object of study of many researchers and considered by National and International Organizations as an age band with priority to actions of promotion, prevention and protection. Many factors contributed to this concern with adolescence, between them, we can emphasize the importance of this populational contingent that represents $30 \%$ of world population, besides the questions of precocious pregnancy, AIDS and drugs. As nurse reseachers, we are interested in experiencing and characterizing quantitatively and qualitatively the scientific production about adolescence in nursing, using as source of data collection the national specific nursing periodicals.Data obtained in this research show that the adolescence theme is not explored by the nursing staff, and the most important aspects are related to sexuality.

KEY WORDS: adolescence, nursing

\section{LA PRODUCCIÓN DEL CONOCIMIENTO SOBRE ADOLESCENCIA EN ENFERMERIA: PERÍODO DE 1983 A 1996}

En los últimos diez años, la adolescencia ha sido objeto de estudio de diversos investigadores y considerada por Organizaciones Nacionales e Internacionales como un grupo etáreo prioritário para acciones de promoción, prevención y protección de la salud. Varios factores contribuyeron para esa preocupación con la adolescencia, entre ellos, podemos destacar la importancia de ese contingente poblacional que representa el 30\% de la población mundial, además de las cuestiones de embarazo precóz, del AIDS y de las drogas. Como Enfermeras Investigadoras, nos interesamos en conocer y caracterizar cuantitativa y cualitativamente la produción científica sobre la adolescencia, realizada por Enfermería, utilizando como fuente de recolección de los dados, las revistas de publicacción periódica del Brasil, en el área especifica de Enfermería. Los datos obtenidos en esse estudio muestran que el area de la adolescencia aún es poco explorada por enfermería y los aspectos más investigados son los relacionados a la sexualidad.

TÉRMINOS CLAVES: adolescencia, enfermería

\section{REFERÊNCIAS BIBLIOGRÁFICAS}

\section{ABERASTURY, A.; KNOBEL, M. Adolescência} normal: um enfoque psicanalítico. 5. ed. Porto Alegre: Artes Médicas, 1986.

02. ARATANGY, L.R. Sexualidade: a difícil arte do encontro. São Paulo: Ática, 1995.

03. BARROSO, C.; BRUSCHINI, C. Sexo e juventude: como discutir a sexualidade em casa e na escola. 4. ed. São Paulo: Cortez, 1991.

04. BRASIL, Ministério da Saúde. Secretaria Nacional de Programas Especiais de Saúde. Programa de saúde do adolescente - PROSAD. Brasília, nov. 1989.

05. CANELLA, P.R.B. Tóxicos na adolescência. In: VITIELlO, N. et al. Adolescência hoje. São Paulo: Roca, 1988.

06. CAVALCANTI, R.C. Adolescência. In: VITIELLO, N. et al. Adolescência hoje. São Paulo: Roca, 1988

07. CONCEIÇÃO, I.S.C. Educação sexual. In: VITIELLO, N. et al. Adolescência hoje. São Paulo: Roca, 1988.

08. ERICKSON, E.H. Identidade, juventude e crise. Rio de Janeiro: Zahar, 1972.
09. FERRIANI, M.G.C. et al. Identificação de alguns conhecimentos sobre drogas em alunos da $3^{\mathrm{a}}$ série do $1^{\circ}$ grau - Relato de uma experiência. Revista Brasileira de Saúde Escolar, v.2, n.2, p. 55 72, 1992.

10. LOPEZ, F.V. et al. Gravidez na adolescência: estudo comparativo. Revista Saúde Pública. São Paulo, v. 23, n. 6, p. 473-7, 1989.

11. OSÓRIO, L.C. Adolescência hoje. 2. ed. Porto Alegre: Artes Médicas, 1992.

12. OUTEIRAL, J.O. Adolescer: estudos sobre adolescência. Porto Alegre: Artes Médicas, 1994.

13. PARKER, R.G. Corpos, prazeres e paixões: a cultura sexual no Brasil contemporâneo. 2. ed. São Paulo: Best Seller, 1991.

14. SEXUALIDADE na adolescência. Revista de Sexologia, n. 1, p. 15-18, 1983.

15. TIBA, I. Puberdade e adolescência: desenvolvimento biopsicossocial. São Paulo: Ágora, 1986.

16. UBEDA, E.M.L. Programa de atendimento à saúde do adolescente: a percepção dos atores sociais envolvidos. Ribeirão Preto, 1996. 206p. Tese (Doutorado) - Escola de Enfermagem de Ribeirão Preto. Universidade de São Paulo.

17. VITIELLO, N. Adolescência hoje. São Paulo: Roca, 1988. 